\title{
A Randomized, Placebo-Controlled Clinical Trial of Tacrolimus Ophthalmic Suspension 0.1\% in Severe Allergic Conjunctivitis
}

\author{
Yuichi Ohashi, ${ }^{1}$ Nobuyuki Ebihara, ${ }^{2}$ Hiroshi Fujishima, ${ }^{3}$ Atsuki Fukushima, ${ }^{4}$ Naoki Kumagai, ${ }^{5}$ Yayoi Nakagawa, ${ }^{6}$ \\ Kenichi Namba, ${ }^{7}$ Shigeki Okamoto, ${ }^{8}$ Jun Shoji, ${ }^{9}$ Etsuko Takamura, ${ }^{10}$ and Kunihiko Hayashi ${ }^{11}$
}

\begin{abstract}
Aims: To examine the efficacy of tacrolimus ophthalmic suspension $0.1 \%$ in treating severe allergic conjunctivitis.

Methods: This was a multicenter, randomized, double-masked, placebo-controlled clinical trial. Fifty-six patients with severe allergic conjunctivitis in whom topical antiallergic agents and corticosteroids had been ineffective were randomized to tacrolimus or placebo treatment. Patients were treated either with tacrolimus or placebo twice-daily for 4 weeks. Severity of objective signs in palpebral and bulbar conjunctiva, limbus, and corneal involvement was assessed using 4 grades. Seven subjective symptoms were evaluated by visual analog scale (VAS) assessment. The primary efficacy endpoint was change in the total score of objective signs at the end of treatment. The secondary efficacy endpoints included change in the score for each objective sign and change in the VAS for each subjective symptom. Safety was assessed based on the severity and the incidence of adverse events.

Results: Mean change from baseline in total score for objective signs was significantly greater in the tacrolimus $(-5.6 \pm 5.1)$ than in the placebo group $(-0.1 \pm 4.5 ; P<0.001)$. Tacrolimus significantly improved giant papillae $(P=0.001)$ and corneal involvement $(P=0.005)$. Five subjective symptoms (itching, discharge, hyperemia, lacrimation, and foreign body sensation) were significantly better in the tacrolimus than in the placebo group. The most frequent treatment-related adverse event in the tacrolimus group was mild ocular irritation upon topical instillation, which was well-tolerated.
\end{abstract}

Conclusion: Tacrolimus ophthalmic suspension $0.1 \%$ is effective in treating severe allergic conjunctivitis.

\section{Introduction}

Cevere allergic ocular diseases, including atopic keraStoconjunctivitis (AKC) and vernal keratoconjunctivitis (VKC), can induce corneal involvement ${ }^{1-3}$ due to inflammatory substances released from giant papillae, leading to corneal shield ulcer or corneal plaque in some cases. These allergic ocular diseases have generally been treated with topical antiallergic agents and/or corticosteroids. For refractory cases, oral or subtarsal corticosteroids are indicated, often resulting in the aggravation of the lesion with tapering or discontinuation. In addition, chronic use of topical corticosteroids may increase intraocular pressure (IOP) and susceptibility to opportunistic infections. A novel treatment

${ }^{1}$ Department of Ophthalmology, University of Ehime School of Medicine, Shizukawa, Toon City Ehime, Japan.

${ }^{2}$ Department of Ophthalmology, Juntendo University school of Medicine, Tokyo, Japan.

${ }^{3}$ Department of Ophthalmology, International University of Health and Welfare, Mita Hospital, Tokyo, Japan.

${ }^{4}$ Department of Ophthalmology, Kochi Medical School, Kochi, Japan.

${ }^{5}$ Kumagai Eye Clinic, Yamaguchi, Japan.

${ }^{6}$ Nakagawa Eye Clinic, Osaka, Japan.

${ }^{7}$ Department of Ophthalmology and Visual Science, Hokkaido University Graduate School of Medicine, Hokkaido, Japan.

${ }^{8}$ Okamoto Eye Clinic, Ehime, Japan.

${ }^{9}$ Department of Ophthalmology, Division of Visual Sciences, Nihon University School of Medicine, Tokyo, Japan.

${ }^{10}$ Department of Ophthalmology, Tokyo Women's Medical University, School of Medicine, Tokyo, Japan.

${ }^{11}$ School of Health Sciences, Gunma University, Gunma, Japan. 
for severe allergic ocular diseases with potent anti-inflammatory effects as well as sufficient safety is thus needed.

Since the report of BenEzra and colleagues, ${ }^{4,5}$ the efficacy of topical cyclosporine against severe allergic ocular diseases has been documented by many investigators. ${ }^{6-11}$ However, high-concentration cyclosporine, in oil-based formulations such as maize, results in intense stinging sensation and blurred vision upon instillation, leading to poor compliance. ${ }^{8}$ To overcome these problems, $0.05 \%$ cyclosporine emulsion was used for AKC and VKC patients and shown to be effective, ${ }^{10,11}$ though it failed to exhibit sufficient clinical efficacy in some steroid-dependent cases. ${ }^{12}$

Tacrolimus is a nonsteroidal immunomodulator isolated from Streptomyces tsukubaensis. ${ }^{13}$ Because of its potent immunosuppressive effect, ${ }^{14}$ topical tacrolimus is expected to exhibit excellent therapeutic efficacy in suppressing abnormal immune responses related to allergic ocular diseases. Based on the efficacy of tacrolimus ointment in treating atopic dermatitis, it has been successfully used at concentrations of $0.02 \%-0.1 \%$ in ointment for various immune-mediated ocular surface disorders. ${ }^{15-20}$ However, the efficacy of tacrolimus eye drops has not been prospectively evaluated in a randomized controlled trial. We conducted a placebocontrolled trial to evaluate the efficacy of tacrolimus ophthalmic suspension $0.1 \%$ in patients with severe allergic conjunctivitis refractory to conventional treatment.

\section{Methods}

\section{Study design}

This was a multicenter, randomized, double-masked, parallel-group, placebo-controlled clinical trial performed to compare tacrolimus ophthalmic suspension $0.1 \%$ and vehicle control placebo (ophthalmic solution free of tacrolimus). Tacrolimus ophthalmic suspension $0.1 \%$ was formulated to be an isotonic aqueous suspension using polyvinyl alcohol as dispersive agents. It is preserved with benzalkonium chloride and submicron size of tacrolimus particle was suspended in it. Dose of tacrolimus was based on the results from dose-ranging study. In that trial, tacrolimus ophthalmic suspension $0.01 \%, 0.03 \%$, and $0.1 \%$ were tested. As a result, $0.1 \%$ showed stronger improvement and similar safety profile compared with $0.01 \%$ and $0.03 \%$. Therefore, we chose $0.1 \%$ as an optimal dose (Astellas Pharma Inc., Japan, 2003). Patients were enrolled at 14 investigational institutions (Appendix) between February and September 2004.

\section{Patients}

Male and female patients 6 years of age or older with severe allergic conjunctivitis (AKC and VKC) refractory to topical antiallergic agents and corticosteroids with moderate to severe giant papillae were eligible. Patients who received systemic administration or subconjunctival injection of corticosteroids, and/or ophthalmic or systemic administration of immunosuppressants within 2 weeks prior to the study; underwent cryosurgery or surgical excision of giant papillae within 4 weeks prior to the study; were receiving desensitization therapy or immune modulation therapy; had infectious eye disease; were pregnant, lactating, or planning pregnancy during the study period; exhibited drug hypersensitivity; were diabetic; or had cardiac, renal, hepatic, or pancreatic disease were excluded.

Before this study, the risks and benefits expected from participation were fully explained to all patients, and informed consent was obtained from each participant. This study was conducted in compliance with Good Clinical Practice and the Declaration of Helsinki. Before the study began, implementation of it was approved by the site Institutional Review Board.

\section{Study procedure}

Eligible patients were randomly allocated to the tacrolimus or placebo group by the minimization method with assignment factors of diagnosis of allergic conjunctivitis and participating medical institutions (implemented with Visual Basic Version 6.0, Microsoft). Patients were instructed to instill a single drop of either tacrolimus or placebo twicedaily for 4 weeks. Study drugs vials were covered by opaque film for masking. If topical corticosteroids, vasoconstrictors, and/or nonsteroidal anti-inflammatory agents were used before study entry, they were discontinued and switched to study drugs. Concomitant use of topical or systemic antiallergic agents was allowed, but only without dose change during the 4-weeks prior to the study and treatment period. Key codes for study drugs were kept secure by Astellas Pharma Inc. (Tokyo, Japan). These codes were broken after fixation of the database for this trial.

Objective signs and subjective symptoms were observed at baseline (before treatment) and 1,2, and 4 weeks after treatment initiation. Ten objective signs for the palpebral conjunctiva (hyperemia, edema, follicles, papillae, and giant papillae), bulbar conjunctiva (hyperemia and chemosis), limbus (Trantas' dot and edema), and corneal involvement were assessed using 4 grades $(0=$ Normal; $1+=$ Mild; $2+=$ Moderate; or $3+=$ Severe, Table 1$).{ }^{21}$

The severity of subjective symptoms (itching, discharge, hyperemia, lacrimation, eye pain, foreign body sensation, and photophobia) was assessed by visual analog scale (VAS). The VAS scored $0 \mathrm{~mm}$ (none) to $80 \mathrm{~mm}$ (very severe). Ocular safety was assessed based on changes in visual acuity, IOP, pupil diameter, and clinical findings for the iris, lens, anterior chamber, and fundus. Laboratory examinations including hematological analysis, blood biochemical tests, and urinalysis were conducted.

The primary efficacy endpoint was change from baseline in total score for objective signs at end of treatment (at 4 weeks after treatment initiation or final observation if treatment was discontinued before week 4). Change from baseline in total score for objective signs at each study visit, change in score for each objective sign, and change in VAS score for symptoms were secondary efficacy endpoints. Safety was assessed based on the severity and incidence of adverse events.

\section{Statistical analysis}

The 2-sided 5\% significance level was applied to statistical hypothesis testing, with confidence intervals (CIs) given as 2-sided $95 \%$ CIs.

Patients who met the eligibility criteria, had at least one dose of study drug, and had at least one efficacy assessment after baseline were included in the efficacy analysis. For each 
Table 1. Grading Scales for Objective Clinical Signs

\begin{tabular}{|c|c|c|}
\hline Signs & Score & Definition \\
\hline \multicolumn{3}{|l|}{ Palpebral conjunctiva } \\
\hline \multirow[t]{4}{*}{ Hyperemia } & 3 & Impossible to distinguish individual blood vessels \\
\hline & 2 & Dilatation of many vessels \\
\hline & 1 & Dilatation of several vessels \\
\hline & 0 & None \\
\hline \multirow[t]{4}{*}{ Edema } & 3 & Diffuse edema with opacity \\
\hline & 2 & Thinner diffuse edema \\
\hline & 1 & Slight edema \\
\hline & 0 & None \\
\hline \multirow[t]{4}{*}{ Follicles } & 3 & 20 or more follicles \\
\hline & 2 & 10-19 follicles \\
\hline & 1 & 1-9 follicles \\
\hline & 0 & None \\
\hline \multirow[t]{4}{*}{ Papillae } & 3 & Papillae size: $0.6 \mathrm{~mm}$ or more \\
\hline & 2 & Papillae size: $0.3-0.5 \mathrm{~mm}$ \\
\hline & 1 & Papillae size: $0.1-0.2 \mathrm{~mm}$ \\
\hline & 0 & None \\
\hline \multirow{4}{*}{$\begin{array}{l}\text { Giant papillae (papillae } \\
\text { size } \geq 1 \mathrm{~mm} \text { ) }\end{array}$} & 3 & Elevated papillae in $1 / 2$ or more of the upper palpebral conjunctiva \\
\hline & 2 & Elevated papillae in $<1 / 2$ of the upper palpebral conjunctiva \\
\hline & 1 & Flat papillae \\
\hline & 0 & None \\
\hline \multicolumn{3}{|l|}{ Bulbar conjunctiva } \\
\hline \multirow[t]{4}{*}{ Hyperemia } & 3 & Diffuse dilated blood vessels over the entire bulbar conjunctiva \\
\hline & 2 & Dilatation of many vessels \\
\hline & 1 & Dilatation of several vessels \\
\hline & 0 & None \\
\hline \multirow[t]{4}{*}{ Edema } & 3 & Bullous edema \\
\hline & 2 & Thinner diffuse edema \\
\hline & 1 & Localized edema \\
\hline & 0 & None \\
\hline \multicolumn{3}{|l|}{ Limbus } \\
\hline \multirow[t]{4}{*}{ Trantas' dot } & 3 & 9 or more dots \\
\hline & 2 & $5-8$ dots \\
\hline & 1 & $1-4$ dots \\
\hline & 0 & None \\
\hline \multirow[t]{4}{*}{ Swelling } & 3 & Found in $2 / 3$ or more of the limbal circumference \\
\hline & 2 & Found in $1 / 3$ to $<2 / 3$ of the limbal circumference \\
\hline & 1 & Found in $<1 / 3$ of the limbal circumference \\
\hline & 0 & None \\
\hline \multirow[t]{4}{*}{ Corneal epithelial signs } & 3 & Shield ulcer or corneal erosion \\
\hline & 2 & Exfoliation superficial punctate keratitis \\
\hline & 1 & Superficial punctate keratitis \\
\hline & 0 & None \\
\hline
\end{tabular}

patient, the eye with higher total score for clinical findings was selected for efficacy assessment. For the primary endpoint, change from baseline in total score for objective clinical signs at end of treatment, the 2 groups were compared by 2 -sample $t$-test. For the secondary endpoints, the 2 groups were compared by 2-sample $t$-test or Mann-Whitney $U$-test. Patients who had at least one dose of study drug and had safety assessment after baseline were included in the safety analysis. Safety assessments were performed based on the incidence of adverse events using Fisher's exact test.

A sample size of 25 patients per group was calculated to detect a mean difference of 5.0 for the primary endpoint with $80 \%$ power and $\alpha=0.05$ (2-sided) assuming a standard deviation (SD) of 6.1 .
Statistical analysis was performed with SAS Version 6.12 and Version 8.2 (SAS Institute, NY).

\section{Results}

\section{Patient disposition and demographics}

Of the 56 patients with severe allergic conjunctivitis (41 AKC and $15 \mathrm{VKC}$ ), 28 each were randomly allocated to the tacrolimus and placebo groups (21 AKC and 7 VKC for the tacrolimus group and $20 \mathrm{AKC}$ and $8 \mathrm{VKC}$ for the placebo group). All AKC patients had atopic dermatitis. Male patients predominated, accounting for $89.3 \%$ of patients $(25 / 28)$ in both groups. Mean $( \pm S D)$ age was $17.9 \pm 9.1$ years 
in the tacrolimus and $15.2 \pm 8.1$ years in the placebo group. Mean total score for objective clinical signs at baseline was $15.3 \pm 4.9$ in the tacrolimus and $17.1 \pm 4.5$ in the placebo group. All patients had received topical antiallergic agents (sodium cromoglicate, amlexanox, ketotifen fumarate, pemirolast potassium, ibudilast, and/or levocabastine hydrochloride) within 4 weeks prior to treatment, and continued to use them during the treatment period, except one patient in the tacrolimus group. Twenty-two patients (78.6\%) each in the tacrolimus and placebo groups had been treated with corticosteroid eye drops including fluorometholone, betamethasone, or dexamethasone before the study.

Treatment with study drugs was discontinued for one patient in the tacrolimus group due to aggravation of allergic conjunctivitis with adverse event, while 5 patients in the placebo group were withdrawn from the study due to aggravation of allergic conjunctivitis and one patient due to an adverse event. All patients were included in safety and efficacy assessments.

\section{Outcome measures}

Total score for objective signs in the tacrolimus group steadily decreased from weeks 1 through 4 . Mean change from baseline $( \pm \mathrm{SD})$ in total score for objective signs at end of treatment was $-5.6 \pm 5.1$ in the tacrolimus and $-0.1 \pm 4.5$ in the placebo group, and significant improvement in the former $(P<0.001)$ was shown in the primary analysis. The difference between 2 groups was -5.5 , and CI ranged from -8.1 to -2.9 . The mean changes from baseline in AKC and VKC patients in the tacrolimus group were lower than in the placebo group (Fig. 1).
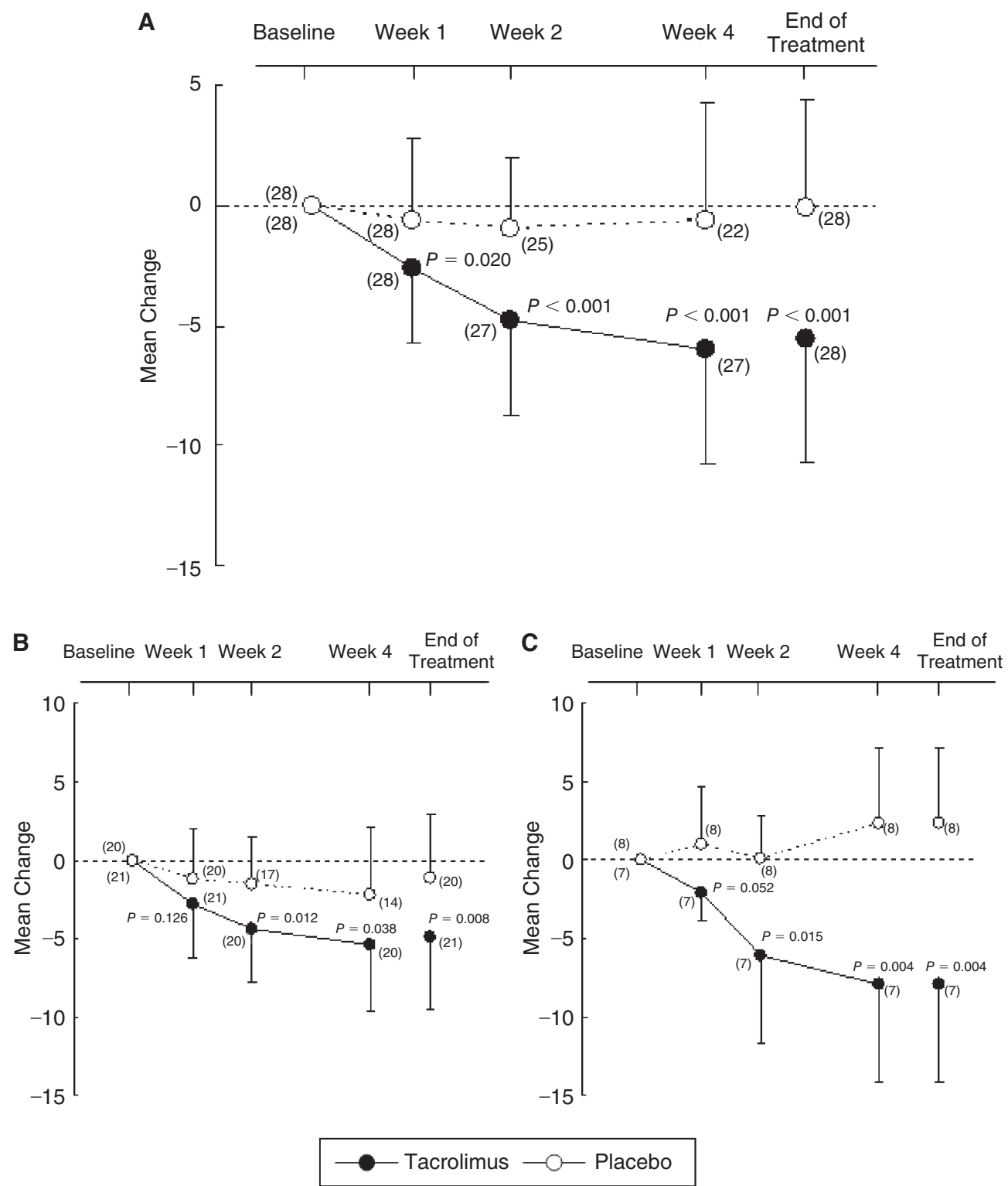

FIG. 1. (A) Mean change from baseline in total score for objective signs. (B) Mean change from baseline in total score for objective signs in atopic keratoconjunctivitis (AKC) patients. (C) Mean change from baseline in total score for objective signs in vernal keratoconjunctivitis (VKC) patients. Significance of differences was evaluated by 2-sample $t$-test compared with placebo group. ( ): patient number. Vertical bars: standard deviation. 
Figure 2 shows changes in distribution of severity of giant papillae, corneal involvement, palpebral conjunctival hyperemia, and bulbar conjunctival hyperemia. Treatment with tacrolimus yielded significantly greater improvement in giant papillae from week 1 through week 4 . The proportions of patients with no or mild giant papillae were $60.7 \%(17 / 28)$ in the tacrolimus and $10.7 \%(3 / 28)$ in the placebo group at end of treatment. Corneal involvement was also significantly improved in the tacrolimus group compared with the placebo group from week 1 through week 4 . No corneal epithelial disturbance was observed in $40.0 \%$ (10/25) of patients in the tacrolimus and 7.7\% (2/26) in the placebo group at end of treatment. Representative photographs of changes in giant papillae and corneal involvement with tacrolimus are shown in Figure 3. Palpebral and bulbar conjunctival hyperemias were improved with time from week 1 to week 4 as giant papillae and corneal involvement. Palpebral conjunctival edema $(P=0.006)$, follicles $(P=0.001)$, and limbal edema $(P=0.033)$ were also significantly improved in the tacrolimus group compared with the placebo group at week 4 .

On VAS assessment, 5 of 7 symptoms (itching, discharge, hyperemia, lacrimation, and foreign body sensation) were
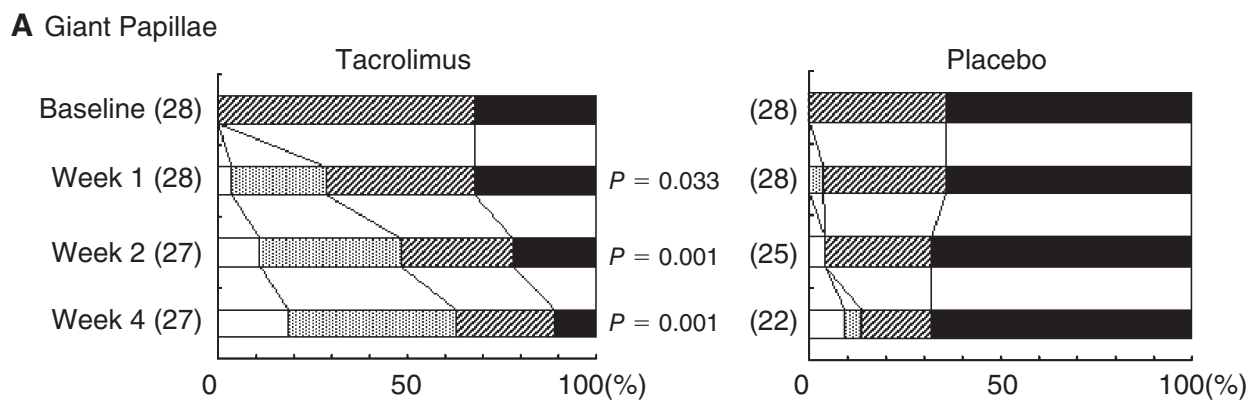

B Corneal Involvement
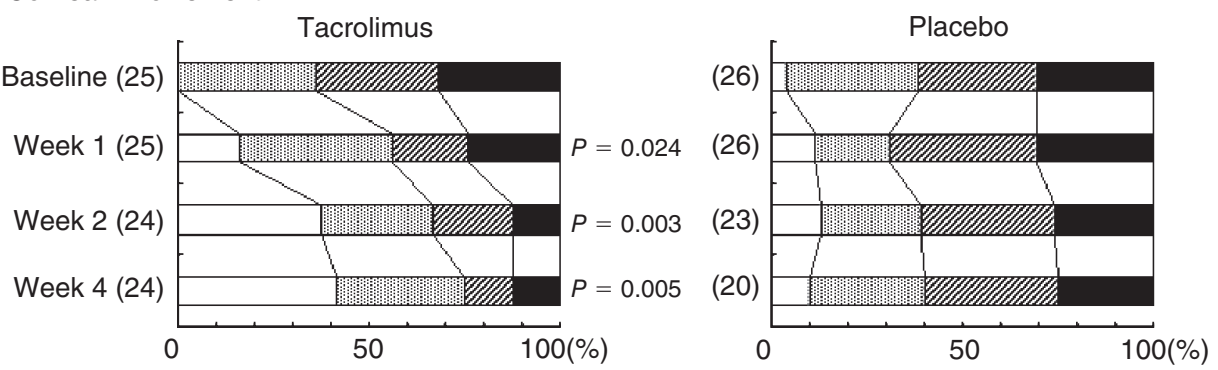

C Palpebral Conjunctival Hyperemia
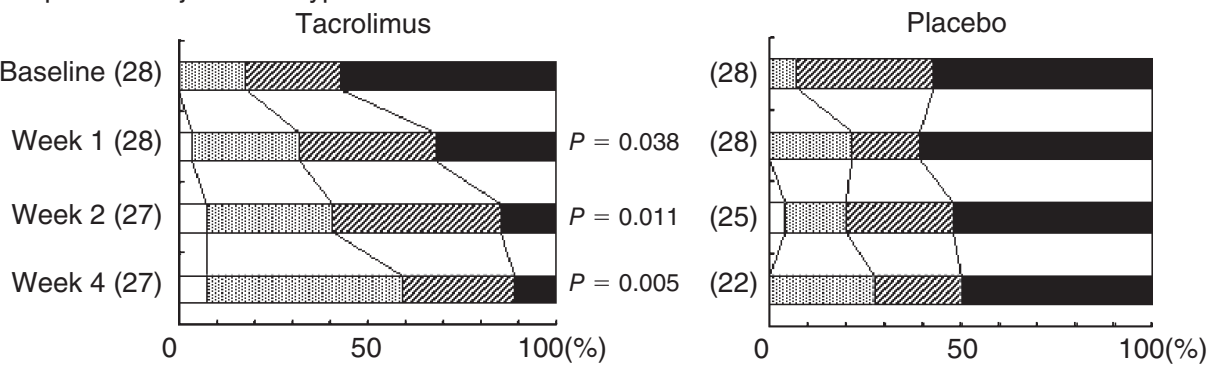

D Bulbar Conjunctival Hyperemia
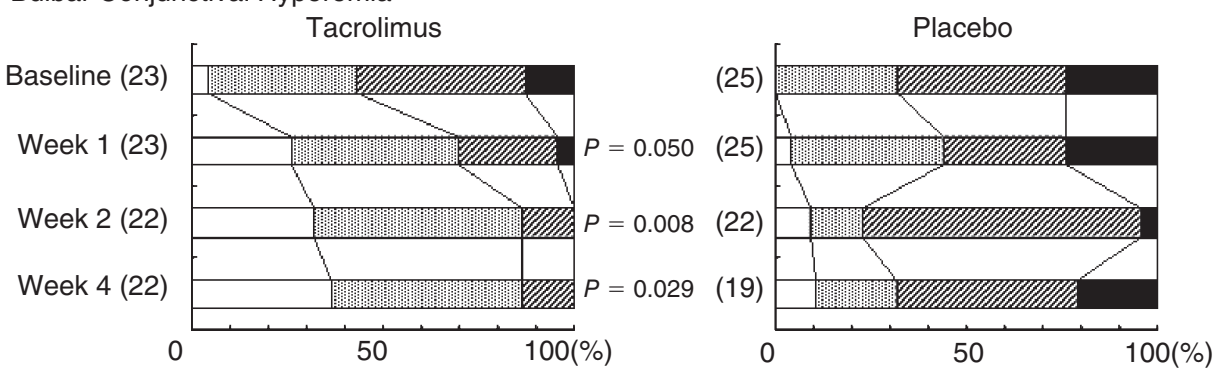

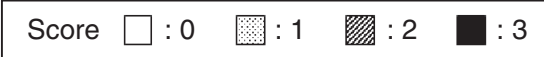

FIG. 2. Distributions of scores for giant papillae, corneal involvement, palpebral conjunctival hyperemia, and bulbar conjunctival hyperemia. Significance of differences was evaluated by Mann-Whitney $U$-test for change in score compared with placebo group. ( ): patient number. 

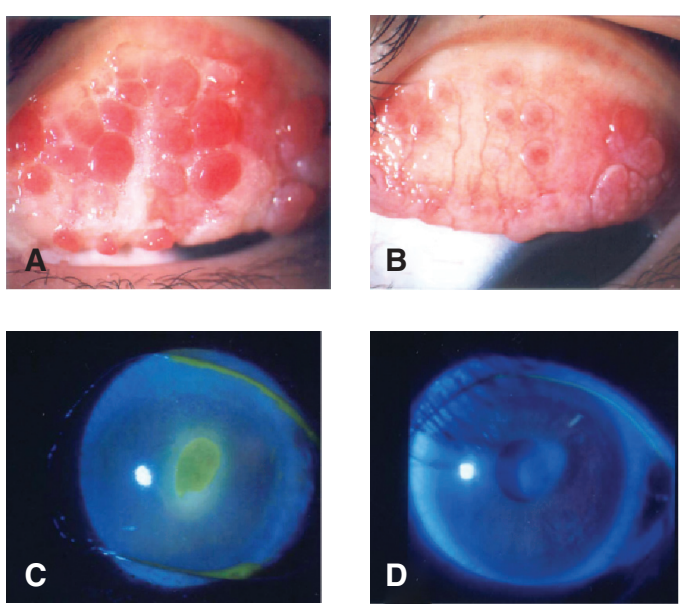

FIG. 3. (A) Giant papillae at baseline in a 9-year-old male vernal keratoconjunctivitis (VKC) patient (case A). (B) After 4-week treatment with tacrolimus ophthalmic suspension in case A. Most giant papillae became flat (score 1+) and less inflamed. (C) Shield ulcer at baseline in 28-year-old female atopic keratoconjunctivitis (AKC) patient (case B). (D) After 4-week treatment with tacrolimus ophthalmic suspension in case B. Shield ulcer disappeared. significantly improved in the tacrolimus group compared with the placebo group at end of treatment (Fig. 4).

In assessment of visual acuity, IOP, pupil diameter, and clinical findings for the iris, lens, anterior chamber, and fundus, hematological analysis, blood biochemical tests, and urinalysis, no abnormal change related to test drugs was observed. No serious adverse events were reported during the study. The incidence of treatment-related adverse events was significantly higher $(P=0.044)$ in the tacrolimus group $(46.4 \%[13 / 28])$ than in the placebo group $(17.9 \%$ [5/28]). The most frequent treatment-related adverse event in the tacrolimus group was mild ocular irritation (42.9\% [12/28]). As ocular infections, there was one case of suspected herpetic keratitis in the tacrolimus group and one of hordeolum in the placebo group. In the tacrolimus group, one case of throat irritation was observed.

\section{Discussion}

The efficacy of topical tacrolimus treatment for severe allergic ocular diseases has recently been intensively studied by many investigators. For example, Vichyanond and coauthors reported marked clinical responses in $10 \mathrm{VKC}$ patients with $0.1 \%$ tacrolimus ointment. ${ }^{15}$ Joseph and coauthors reported that one patient with severe AKC responded

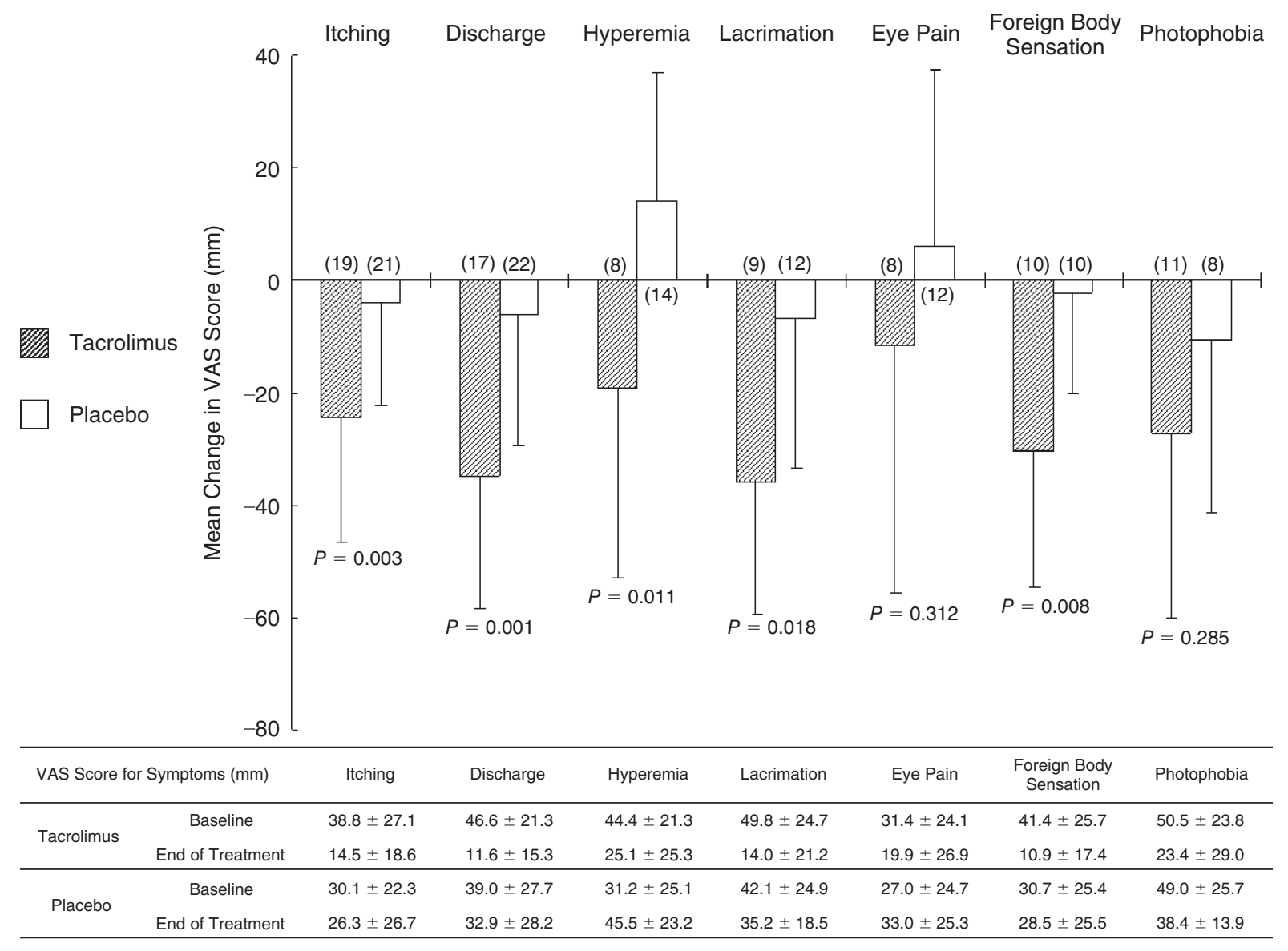

FIG. 4. Visual analog scale (VAS) for each subjective symptom. The figure shows mean (SD) change from baseline in each symptom at end of treatment. Significance of differences was evaluated by 2-sample $t$-test compared with placebo group. Range for each symptom: 0-80 mm. ( ): patient number. Vertical bars: standard deviation. 
dramatically to $0.03 \%$ tacrolimus ointment. ${ }^{16}$ Miyazaki and coauthors reported that $1 \mathrm{VKC}$ and $5 \mathrm{AKC}$ patients who had been refractory to conventional treatment were successfully treated with $0.02 \%$ tacrolimus ointment. ${ }^{19}$ Nevertheless, the efficacy of tacrolimus requires evaluation in larger numbers of patients in a randomized, controlled trial.

Consistent with previous reports, we found that tacrolimus ophthalmic suspension $0.1 \%$ twice-daily yielded marked improvement in objective signs in severe allergic conjunctivitis refractory to conventional treatment. Improvement was observed as early as 1 week after initiation of treatment and continued up to week 4 . Similar findings were observed in the subgroups of AKC and VKC patients. Tacrolimus also improved symptoms; the 2 most frequent symptoms, itching and discharge, were effectively suppressed by it. These findings verified the excellent efficacy of tacrolimus ophthalmic suspension in treating severe allergic conjunctivitis.

Corneal scarring due to severe, prolonged epithelial disturbance such as shield ulcer is known to impair visual acuity in patients with severe allergic conjunctivitis. ${ }^{1-3}$ In patients with conjunctival proliferative change, suppression of inflammation of giant papillae is essential for successful management, since corneal epithelial disturbance results from cytotoxic effects of inflammatory molecules released from lesions at the inflamed palpebral conjunctiva. With topical tacrolimus, giant papillae became less inflamed and flattened, with profound decrease in ropy discharge, finally resulting in inflamed flat giant papillae in about $60 \%$ of patients and disappearance of giant papillae in $20 \%$ of patients. Improvement of corneal involvement was rapid as well, and synchronized with suppression of inflammatory response of giant papillae. Tacrolimus ophthalmic suspension significantly improved the score for corneal epithelial disturbance including shield ulcers without affecting visual acuity. However, moderate to severe giant papillae (score $\geq 2+$ ) persisted in some patients even after treatment with topical tacrolimus for 4 weeks, suggesting that longer treatment might have been needed to improve efficacy.

One of the expected advantages of nonsteroidal immunomodulator is safe tapering or discontinuation topical corticosteroids. Daniell and colleagues reported that lowconcentration topical cyclosporine was not beneficial as a steroid-sparing agent in steroid-dependent allergic conjunctivitis. ${ }^{12}$ In the present study, patients treated with topical corticosteroids with insufficient effects could be switched to topical tacrolimus without rebound phenomenon. These findings suggest the usefulness of topical tacrolimus $0.1 \%$ as a steroid-sparing substitute for treating severe allergic conjunctivitis due to its potent immunosuppressive effect, which is up to 100 times stronger than that of cyclosporine. ${ }^{14}$ To reach more objective conclusions regarding steroidsparing effects of topical tacrolimus, better planned clinical studies are needed.

Tacrolimus ophthalmic suspension $0.1 \%$ was welltolerated. The most frequent tacrolimus-related adverse event was ocular irritation. In the tacrolimus group, ocular irritation occurred in 12 of 28 patients $(42.9 \%)$, but no severe ocular irritation was observed. Opportunistic infection can often occur with use of immunosuppressants or immunomodulators. In the tacrolimus group, one AKC patient developed corneal lesion suspected to be herpes simplex virus infection, although precise virological diagnosis was not performed. Therefore, close follow-up of patients receiving topical tacrolimus, particularly those with atopic dermatitis, is thus always needed, since they are susceptible to herpes simplex virus infection. On the other hand, the incidence of neither herpes simplex virus nor varicella zoster infection was found to be increased with use of tacrolimus ointment in patients with atopic dermatitis in long-term follow-up studies. ${ }^{22,23}$ Whether topically instilled tacrolimus elicits reactivation of HSV requires further observation.

In conclusion, tacrolimus ophthalmic suspension $0.1 \%$ was proven effective in improving objective clinical signs and subjective symptoms of severe allergic conjunctivitis refractory to topical antiallergic agents and corticosteroids compared with placebo. Despite mild irritation upon topical instillation, tacrolimus ophthalmic suspension $0.1 \%$ is considered feasible for treatment of severe allergic conjunctivitis.

\section{Acknowledgment}

This study was supported by Astellas Pharma Inc., Japan, and Senju Pharmaceutical Co., Ltd., Japan.

\section{Author Disclosure Statement}

Dr. Ohashi was contracted with Astellas Pharma Inc. and Senju Pharmaceutical Co., Ltd. as medical adviser for this clinical trial. Dr. Kumagai and Dr. Nakagawa were contracted with Astellas Pharma Inc. as medical adviser. Dr. Hayashi was contracted with Astellas Pharma Inc. as biostatistical advisor. Other authors have no commercial or proprietary interest in the products or the company mentioned in this article.

\section{References}

1. Bonini, S., Bonini, S., Lambiase, A., et al. Vernal keratoconjunctivitis revisited: a case series of 195 patients with long-term followup. Ophthalmology. 107:1157-1163, 2000.

2. Tabbara, K.F. Ocular complications of vernal keratoconjunctivitis. Can. J. Ophthalmol. 34:88-92, 1999.

3. Foster, C.S., and Calonge, M. Atopic keratoconjunctivitis. Ophthalmology. 97:992-1000, 1990.

4. BenEzra, D., Pe'er, J., Brodsky, M., et al. Cyclosporine eyedrops for the treatment of severe vernal keratoconjunctivitis. Am. J. Ophthalmol. 101:278-282, 1986.

5. BenEzra, D., Matamoros, N., and Cohen, E. Treatment of severe vernal keratoconjunctivitis with cyclosporine A eyedrops. Transplant. Proc. 20:644-649, 1988.

6. Secchi, A.G., Tognon, M.S., and Leonardi, A. Topical use of cyclosporine in the treatment of vernal keratoconjunctivitis. Am. J. Ophthalmol. 110:641-645, 1990.

7. Bleik, J.H., and Tabbara, K.F. Topical cyclosporine in vernal keratoconjunctivitis. Ophthalmology. 98:1679-1684, 1991.

8. Hingorani, M., Moodaley, L., Calder, V.L., et al. A randomized, placebo-controlled trial of topical cyclosporin A in steroid-dependent atopic keratoconjunctivitis. Ophthalmology. 105:1715-1720, 1998.

9. Pucci, N., Novembre, E., Cianferoni, A., et al. Efficacy and safety of cyclosporine eyedrops in vernal keratoconjunctivitis. Ann. Allergy Asthma Immunol. 89:298-303, 2002. 
10. Akpek, E.K., Dart, J.K., Watson, S., et al. A randomized trial of topical cyclosporin $0.05 \%$ in topical steroidresistant atopic keratoconjunctivitis. Ophthalmology. 111:476482, 2004.

11. Ozcan, A.A., Ersoz, T.R., and Dulger, E. Management of severe allergic conjunctivitis with topical cyclosporin a $0.05 \%$ eyedrops. Cornea. 26:1035-1038, 2007.

12. Daniell, M., Constantinou, M., Vu, H.T., et al. Randomised controlled trial of topical ciclosporin A in steroid dependent allergic conjunctivitis. Br. J. Ophthalmol. 90:461-464, 2006.

13. Kino, T., Hatanaka, H., Hashimoto, M., et al. FK-506, a novel immunosuppressant isolated from a Streptomyces. I. Fermentation, isolation, and physico-chemical and biological characteristics. J. Antibiot. 40:1249-1255, 1987.

14. Kino, T., Hatanaka, H., Miyata, S., et al. FK-506, a novel immunosuppressant isolated from a Streptomyces. II. Immunosuppressive effect of FK-506 in vitro. J. Antibiot. 40:1256-1265, 1987.

15. Vichyanond, P., Tantimongkolsuk, C., Dumrongkigchaiporn, P., et al. Vernal keratoconjunctivitis: Result of a novel therapy with $0.1 \%$ topical ophthalmic FK-506 ointment. J. Allergy Clin. Immunol. 113:355-358, 2004.

16. Joseph, M.A., Kaufman, H.E., and Insler, M. Topical tacrolimus ointment for treatment of refractory anterior segment inflammatory disorders. Cornea. 24:417-420, 2005.

17. Virtanen, H.M., Reitamo, S., Kari, M., et al. Effect of $0.03 \%$ tacrolimus ointment on conjunctival cytology in patients with severe atopic blepharoconjunctivitis: a retrospective study. Acta. Ophthalmol. Scand. 84:693-695, 2006.

18. Kymionis, G.D., Goldman, D., Ide, T., et al. Tacrolimus ointment $0.03 \%$ in the eye for treatment of giant papillary conjunctivitis. Cornea. 27:228-229, 2008.
19. Miyazaki, D., Tominaga, T., Kakimaru-Hasegawa, A., et al. Therapeutic effects of tacrolimus ointment for refractory ocular surface inflammatory diseases. Ophthalmology. 115:988-992.e5, 2008.

20. Attas-Fox, L., Barkana, Y., Iskhakov, V., et al. Topical tacrolimus $0.03 \%$ ointment for intractable allergic conjunctivitis: an openlabel pilot study. Curr. Eye Res. 33:545-549, 2008.

21. Uchio, E., Kimura, R., Migita, H., et al. Demographic aspects of allergic ocular diseases and evaluation of new criteria for clinical assessment of ocular allergy. Graefes Arch. Clin. Exp. Ophthalmol. 246:291-296, 2008.

22. Kang, S., Lucky, A.W., Pariser, D., et al. Long-term safety and efficacy of tacrolimus ointment for the treatment of atopic dermatitis in children. J. Am. Acad. Dermatol. 44:S58-S64, 2001.

23. Hanifin, J.M., Paller, A.S., Eichenfield, L., et al.; US Tacrolimus Ointment Study Group. Efficacy and safety of tacrolimus ointment treatment for up to 4 years in patients with atopic dermatitis. J. Am. Acad. Dermatol. 53:S186-S194, 2005.

Received: August 12, 2009

Accepted: December 23, 2009

Address correspondence to:

Prof. Yuichi Ohashi

Department of Ophthalmology

University of Ehime School of Medicine

Shizukawa

Toon City Ehime, 7910295

Japan

E-mail: ohashi@m.ehime-u.ac.jp 


\section{Appendix: Members of the Tacrolimus Study Group}

\section{Medical Adviser:}

Yuichi Ohashi, University of Ehime School of Medicine, Ehime, Japan.

Naoki Kumagai, Kumagai Eye Clinic, Yamaguchi, Japan.

Yayoi Nakagawa, Nakagawa Clinic, Osaka, Japan.

\section{Coordinating Investigator:} Japan.

Shigeaki Ohno, Hokkaido University Hospital, Hokkaido,

\section{Study Investigators:}

Kenichi Namba, Masahiro Muramatsu, and Satomi Takahashi, Hokkaido University Hospital, Hokkaido;

Tadahiko Tsuru, Hiroto Obata, Taeko Inoki, Shinsuke Aoki, and Yoshiki Nakano, Jichi Medical University Hospital, Tochigi;

Hiroshi Fujishima, Yoji Takano, and Yoshiyuki Satake, Tokyo Dental College Ichikawa General Hospital, Chiba;

Sadao Hori, Etsuko Takamura, Keiko Nomura, and Hiroko Araki, Tokyo Women's Medical University Hospital, Tokyo;

Akira Murakami, Nobuyuki Ebihara, Hiroshi Toshida, and Hidenori Sasaki, Juntendo University Hospital, Tokyo;

Noriko Inada, Jun Shoji, and Keiko Saito, Nihon University Itabashi Hospital, Tokyo;
Yuuta Sano, Genichiro Takahashi, Yukiko Nagai, and Takuya Shiba, The Jikei University Hospital, Tokyo;

Misaki Ishioka and Kazumi Fukagawa, Ryogoku Eye Clinic, Tokyo;

Nobuyuki Ooguro, Hitoshi Watanabe, Koji Nishida, and Naoyuki Maeda, Osaka University Hospital, Osaka;

Shigeki Okamoto, Naoko Hashimoto, and Chizuko Ide, Kozuka Eye Center, Ehime;

Atsuki Fukushima, Kochi Medical School Hospital, Kochi;

Kazunori Miyata, Ryohei Nejima, and Takashi Miyai, Miyata Eye Hospital, Miyazaki;

Taiji Sakamoto, Shozo Sonoda, Kumiko Nakao, and Eisuke Uchino, Kagoshima University Medical and Dental Hospital, Kagoshima;

Yoshikazu Shimomura, Masahiko Fukuda, Shiro Higaki, and Naoko Taenaka, Kinki University Hospital, Osaka, Japan.

\section{Biostatistical Advisor:}

Kunihiko Hayashi, School of Health Sciences, Gunma University, Gunma, Japan.

\section{Safety Monitoring Committee:}

Yujiro Hujino, Tokyo Kosei Nenkin Hospital, Tokyo, Japan.

Kunio Morozumi, Nagoya Daini Red Cross Hospital, Aichi, Japan. 
